\title{
Fever and a rash
}

\author{
KJM Jeffery, SJ Peacock
}

A 37-year-old man presented with acute confusion following five days of back pain, and two days of fever and diarrhoea. On examination he had a fever of $38.7^{\circ} \mathrm{C}$, jaundice, neck stiffness, conjunctival suffusion, and a purpuric rash (figures 1 and 2). His pulse was 100 beats/min and blood pressure 100/80 $\mathrm{mmHg}$. The heart sounds were normal. He was tachypnoeic with scattered wheezes in the chest. The rest of the examination was unremarkable.

Laboratory results revealed thrombocytopenia, mild disseminated intravascular coagulation, hyponatraemia, marked renal and hepatic impairment, and hypoxia. Cerebrospinal fluid (CSF) white cell count was $150 / \mathrm{mm}^{3}\left(80 \%\right.$ polymorphs), erythrocytes $800 / \mathrm{mm}^{3}$, protein $1.18 \mathrm{~g} / 1$, glucose $2.6 \mathrm{mmol} / 1$ (plasma glucose $6.8 \mathrm{mmol} / \mathrm{l}$ ) with no organisms on Gram stain. CSF culture was negative. Gram staining of an aspirate from the vasculitic lesions showed Gram-positive cocci in clusters. An organism was grown in blood and urine cultures on the following day. A diagnostic procedure was performed.

\author{
John Radcliffe \\ Hospital, \\ Headington, Oxford \\ OX3 9DU, UK \\ PHLS Department of \\ Microbiology \\ KJM Jeffery \\ Adhesion and \\ Infection Laboratory \\ SJ Peacock
}

Correspondence to $\mathrm{Dr}$ KJM Jeffery, Department of Immunology, Imperial College School of

Medicine at St Mary's, Norfolk Place, London W2 1PG, UK

Accepted 30 October 1996

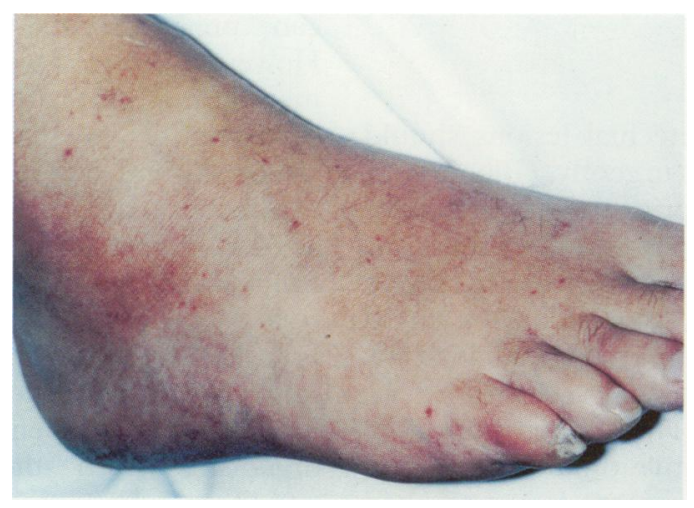

Figure 1 Purpuric rash on right foot

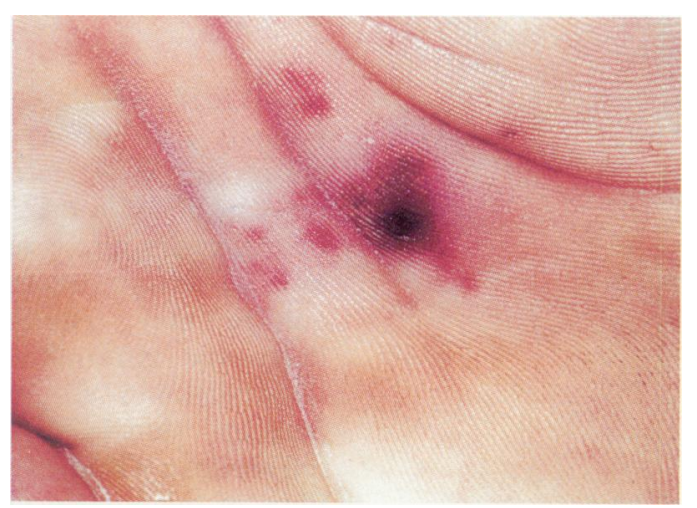

Figure 2 Detail of skin lesion on right palm

\section{Questions}

1 What is the differential diagnosis?

2 What further investigations can be performed to provide rapid diagnostic information? 


\section{Answers}

QUESTION 1

The two most common bacterial infections in the UK associated with a fever and a petechial or purpuric rash are meningococcal septicaemia and meningitis, and Staphylococcus aureus endocarditis. Other infections include disseminated gonococcal disease, and bacteraemic infections with Streptococcus pneumoniae and Capnocytophaga canimorsus (associated with dog bites). In patients with a history of foreign travel other pathogens should be considered: Plasmodium falciparum, Rickettsia rickettsii (Rocky Mountain spotted fever), Rickettsia prowazekii (epidemic/louse-bourne typhus) and Streptobacillus moniliformis (rat-bite fever). Viral illnesses associated with petechial rashes include enteroviruses (especially coxsackievirus A9, echovirus 9), Epstein-Barr virus, cytomegalovirus, atypical measles, and viral haemorrhagic fevers.

The diagnosis in this case was Staphylococcus aureus endocarditis.

\section{QUESTION 2}

Patients with diffuse petechial lesions should always be investigated urgently. Blood cultures, if possible taken prior to administration of antibiotics, are essential, but full identification of an organism grown from a blood culture may require several days. Rapid diagnostic information may be provided by Gram-staining of material aspirated from petechial skin lesions. The demonstration of intracellular Gram-negative diplococci in skin films in meningococcal disease is not affected by antibiotics and can be significantly more sensitive than Gram staining of CSF. ${ }^{1}$ Grampositive cocci may be seen in aspirates from skin lesions associated with $S$ aureus endocarditis, although interpretation should incorporate the possibility of contamination by skin flora, and the aspirate should be cultured to confirm the identity of any micro-organisms seen. Gram films made following aspiration of inflamed joints may similarly provide rapid diagnostic information. CSF Gram stain may be diagnostic in meningococcal disease. The CSF white cell count is also often abnormal in $S$ aureus endocarditis ${ }^{2}$ which may reflect significant intracerebral complications such as meningitis, brain abscess, mycotic aneurysm and cerebral embolism, ${ }^{2,3}$ increasing the importance of computed tomography of the brain prior to lumbar puncture of the obtunded patient. Rapid bacterial antigen testing by latex agglutination of CSF rarely provides additional information to that already obtained by Gram stain. ${ }^{4}$ Polymerase chain reaction amplification of bacterial DNA in appropriate clinical specimens will provide rapid diagnostic information in the future. Echocardiography should be performed in suspected cases of endocarditis. Transoesophageal echocardiography is more sensitive than the transthoracic approach in detecting vegetations, although their absence does not exclude the diagnosis. As indicated above, specific features in the patient's history may suggest other rapid investigations.

\section{Discussion}

$S$ aureus was grown from cultures of blood, urine and aspirates of the petechial lesions. A transthoracic echocardiogram confirmed a mitral valve vegetation. He was managed with appropriate antibiotics and intensive care unit support, including ventilation. Four days after admission he suffered a sub-arachnoid haemorrhage, developed acute hydrocephalus and died.

This case provides a reminder of the difficulty in differentiating $S$ aureus endocarditis from meningococcaemia. ${ }^{5}$ The specificity of symptoms distinguishing between these two conditions may be poor. For example, the headache and obtundation classically associated with meningitis may also accompany $S$ aureus endocarditis, ${ }^{3}$ and both diseases can present with painful swollen joints. However, patients with $S$ aureus endocarditis may have a history of skin sepsis, intravenous drug abuse or intravenous device-related infection, and $50-66 \%$ will have underlying cardiac disease. ${ }^{2,3}$ Examination should include a careful search for skin lesions. Petechial haemorrhages may feature in both meningococcal septicaemia and $S$ aureus endocarditis, but skin sepsis involving pus formation may represent either the initial focus of infection for staphylococcal endocarditis or seeding or organisms from the heart valve to the periphery. All accessible joints should be inspected for evidence of septic arthritis since these provide an additional avenue of investigation and require specific management. The diagnosis of $S$ aureus endocarditis is not excluded by the absence of a cardiac murmur. $^{2,3}$

$S$ aureus endocarditis presenting with features which mimic those of meningococcal septicaemia is rare and, as a result, may initially pass unrecognised. However, there are important implications in reaching the correct diagnosis in view of the difference in management of these two conditions. These include

\section{Summary points}

- $S$ aureus endocarditis may present acutely with a fever and rash mimicking meningococcal septicaemia

- the specificity of symptoms and signs in distinguishing between $S$ aureus endocarditis and meningococcal septicaemia may be poor

- techniques such as Gram staining of aspirates from skin lesions or inflamed joints may provide rapid diagnostic information

- correct diagnosis has implications for management of the patient, including an understanding of potential complications, and choice and duration of antimicrobial therapy 
choice and duration of antimicrobial therapy, and an understanding of the potential complications and their respective treatment. Knowledge of the existence of this clinical entity is the first step to early recognition and appropriate management.

1 Periappuram M, Taylor MRH, Keane CT. Rapid detection of meningococci from petechiae in adults in acute meningococcal infection. F Infect 1995; 31: 201-3.

2 Watanakunakorn C, Tan JS, Phair JP. Some salient features of Staphylococcus aureus endocarditis. Am F Med 1973; 54: 473-81.

3 Watanakunakorn C. Staphylococcus aureus endocarditis at a community teaching hospital, 1980 to 1991 . Arch Intern Med 1994; 154: 2330-5.

\section{Final diagnosis}

\section{Staphylococcus aureus endocarditis}

Keywords: Staphylococcus aureus, endocarditis, meningococcal septicaemia, differential diagnosis

4 Perkins MD, Mirret S, Reller LB. Rapid bacterial antigen detection is not clinically useful. f Clin Microbiol 1995; 33: $1486-91$

5 Murray HW, Tuazon CU, Sheagren JN. Staphylococcal septicaemia and disseminated intravascular coagulation. Staphylococcus aureus endocarditis mimicking meningococcaemia. Arch Intern Med 1977; 137: 844-7.

\title{
A retrocardiac shadow in an immunocompromised patient
}

\author{
FU Huwez, MJ Stewart, AR McPhaden, IS Stewart, SK Naik, D Richens, DJ Wheatley
}

A 53-year-old male ex-smoker underwent orthotopic heart transplantation for severe heart failure following a massive anterior myocardial infarction. Immunosuppression was induced with equine antithymocyte globulin and maintained on cyclosporin A, azathioprine and prednisolone. Virological screen before transplantation revealed positive serology for cytomegalovirus but he was negative for Epstein-Barr virus (EBV). The patient remained well until about two and half years post-transplant when he presented with features of left ventricular failure following symptoms of a chest infection. The latter was treated with two courses of antibiotics in the community prior to his presentation. On examination he was haemodynamically stable and

The Royal Infirmary, Glasgow G31 2ER, UK

Department of Cardiac Surgery

FU Huwez

SK Naik

D Richens

DJ Wheatley

Department of

Medical Cardiology

MJ Stewart

Department of

Pathology

AR McPhaden

Department of

Radiology

IS Stewart

Correspondence to

Dr FU Huwez,

Department of Medicine,

Edgeware General

Hospital, Edgeware,

Middlesex HA8 OAD, UK

Accepted 7 March 1996 afebrile. Electrocardiogram revealed evidence of an acute inferior myocardial infarction which was complicated by heart block, necessitating insertion of a permanent pacemaker. Chest X-ray showed elevation of the left hemi-diaphragm, a small left pleural effusion and a rounded opacity behind the left cardiac border. A contrast computed tomography (CT) scan of the chest was carried out (figure 1).

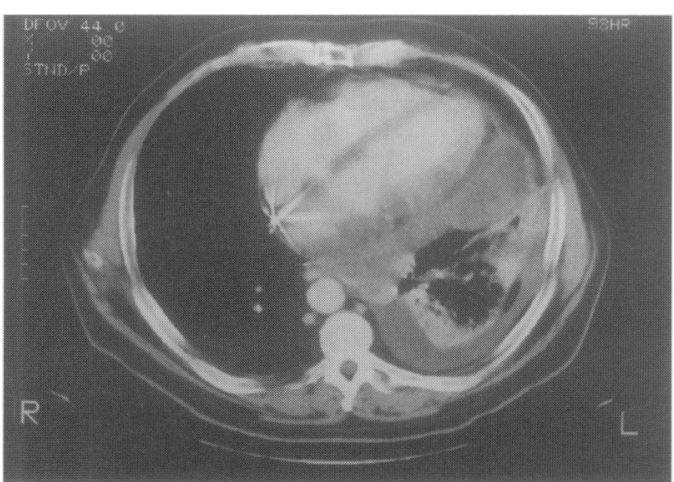

Figure 1 CT scan of the chest

\section{Questions}

1 What are the findings on the CT scan? 2 What further investigations are required ? 3 What is the differential diagnosis? 